\title{
Optimization Design and Calculation of Mooring System Parameters based on Genetic Algorithm
}

\author{
Liang-yu Xia, Li Zhi, Ling Chen and Yue Cheng \\ College of Electrical Engineering \& New Energy, CTGU, Yichang 443002, China
}

\begin{abstract}
Based on the static equilibrium model of mooring system parameters, the multi - objective optimization model is established and the genetic algorithm is introduced as the optimization algorithm, which improves the crossover operator. Taking the example of a single point mooring system, concerned optimization design and calculation has been done. Compared with the general genetic algorithm, the result of this algorithm is superior, significantly improving the stability of the mooring system.
\end{abstract}

\section{Introduction}

The single point mooring system has been widely used in both ship navigation and the comprehensive utilization and development of Marine resources. The small changes of mooring system parameters can greatly affect the stability of the whole system, so a large number of experts have carried out relevant researches. Ming-wu Wang [1] established a two-dimensional model of the underwater buoy system and calculated relevant parameters according to static equilibrium; John [2] used catenary method and polygon approximation method for analysis and calculation; Xing-ping Xu [3] studied single point mooring system from the perspective of dynamics.

Genetic algorithm is a kind of random searching method which is developed by the genetic mechanism of biology. It regards the possible solution in the problem as an individual in the group, and encodes it as a chromosome. Then simulating the biological evolution process, the group receives repeated hybrid operation and chooses the better one continuously, according to the evolution of the biological sector. At the same time, the optimal individual in the optimal group can be found, which is the optimal solution for satisfying the requirements.

\section{Improvement of the genetic algorithm of crossover operators}

The basic genetic algorithm also has many shortcomings, which often can only approach the optimal solution rather than calculate the optimal solution, and sometimes find the local optimal solution. In order to solve these problems, generally beginning with genetic algorithm three basic genetic operators (select operator, crossover operator and mutation operator) of genetic algorithm. In this paper, improved crossover operator's genetic algorithm [4 5] is used to solve the multi-objective optimization problem.

To enhance the diversity of population in the early evolution period, cross-operation between the parent individuals should be expanded to generate a variety of new individuals, which can choose many excellent individuals by copying the operator. However, at the late stage of evolution, with the high frequency of evolution, the solution group gradually approaches the optimal solution. At this point, if we still use a high crossover rate, it will produce a lot of new individuals, which distribute divergently in the entire search space, leading to the low proportion of good individuals in the population. The high rate will delay the convergence process. Therefore, the crossover rate is modified as follows:

$$
p_{c}=p_{c 0}-\frac{d\left(p_{c 0}-p_{c \min }\right)}{D}
$$

Where: $p_{c 0}$-a initial crossover rate; $d$ - the current evolutionary algebra; $D$-the total evolutionary number. It is not difficult to see that with the increase of the iteration times, the crossover rate decreases and the convergence process is accelerated. Genetic algorithm is the search process of every generation based on the adaptability, which is mainly composed of coding mechanism, control parameters, fitness function and genetic operators. The main calculation process of solving the multi-objective optimization problem is as follows: The process is shown in Fig. 1:

a. Initialize the population: randomly generate pop_size feasible chromosomes.

b. Calculate the population fitness: fitness function is used to evaluate the adaptability of each chromosome. In this paper we use the objective function as fitness function, the maximum level of 
fitness means the best individual.

c. Selection process: select one chromosome at a time as a new subgroup. Repeat this process pop_size times to get a new generation.

d. Variation operation: supposing the probability of cross operation is $P_{m}$. Randomly select a chromosome $C_{i}$ as the father of the mutation and randomly generate real numbers which belong to $[0,1]$. If $s<P_{m}$, a mutation in one of the genes of the chromosome is selected randomly.

\section{Start}

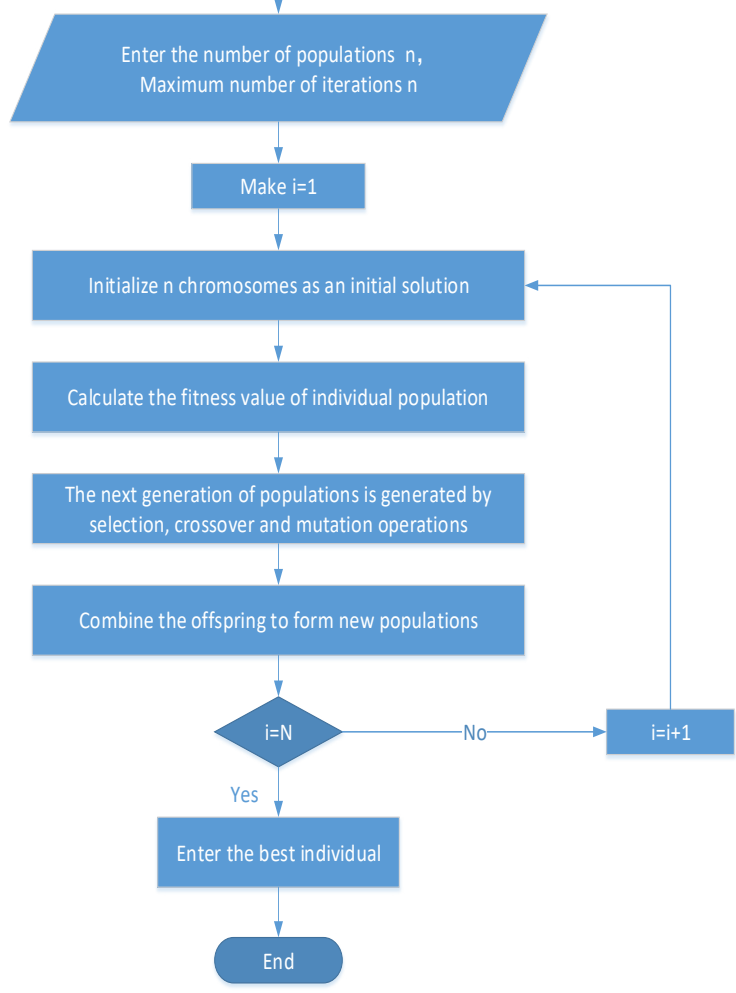

Figure 1: Flow chart of genetic algorithm for improved crossover operator

\section{Static analysis of mooring system}

The single point mooring system consists of steel pipe, steel bucket, heavy ball, electric welding anchor chain and special anti-drag and shifting anchor. Its schematic diagram is shown in Figure 2. This paper uses a polygon approximation method of concentrated mass [3] to conduct two-dimensional static analysis of mooring system.

\subsection{Buoy force analysis}

The equation of equilibrium is as follows:

$$
\left\{\begin{array}{l}
\text { horizontal direction: } T \cos \theta_{i}=f \\
\text { vertical direction: } T \sin \theta_{i}=F_{\text {buoyancy }}-G_{\text {buoy }}
\end{array}\right.
$$

Where: $F_{\text {buyancy }}$-the buoyancy of the buoy in the sea, $G_{\text {buoy }}$-the gravity of the buoy itself, $T$-the tension of the steel tube, $f$-the resistance of the float when the buoy is swimming. The buoyancy is $F_{\text {buovancy }}=\rho_{\text {sea }} \mathrm{g} \times \pi(D / 2)^{2} h$, resistance is $f=(1 / 2) \rho_{\text {sea }} C_{D} S V|V|, \theta$ is the angle between the tension $T$ and the horizontal direction, $D$ is the diameter of the buoy, $C_{D}$ is the resistance coefficient of the fluid to the buoy, $S$ is the horizontal wetting area of the buoy, $V$ is the unidirectional flow rate that represents the fluid at the buoy.

\subsection{Force Analysis of Steel Pipe and Steel}

The equation can be:

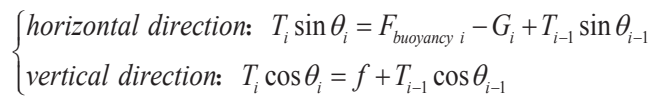

Where: $F_{\text {buoyancy } i}$-buoyancy of the steel pipe in the water, $f$-the water resistance, $G_{i}$-the gravity of the steel tube, $T_{i}$-the force of the previous steel tube, $T_{i-1}$-the force of the next steel pipe. And we can get

$$
T_{i}=\sqrt{\left(F_{\text {buoyancy }_{i}}-G_{i}+T_{i-1} \sin \theta_{i-1}\right)^{2}+\left(f+T_{i-1} \cos \theta_{i-1}\right)^{2}},
$$

$\theta_{i}=\arctan \frac{F_{\text {buoyancy } i}-G_{i}+T_{i-1} \sin \theta_{i-1}}{f+T_{i-1} \cos \theta_{i-1}}$ by derivation. It can be seen from the derivation formula that if the former tension $T_{i-1}$ is given, the following tension $T_{i}$ can be calculated, and the steps in the middle can also be calculated by this method. The force analysis of the steel drums is similar to that of steel pipes, and the difference is that the gravity of the steel drum will be combined with the gravity of the steel ball.

\subsection{Stress analysis of the link}

As the volume of the link is very small, it can be considered without buoyancy and hydrodynamic effect, that is, only bears its own gravity and the force of the previous link and the chain, we can get the equilibrium equation:

$$
\left\{\begin{array}{l}
\text { horizontal direction: } T_{i} \sin \theta_{i}=G_{i}+T_{i-1} \sin \theta_{i-1} \\
\text { vertical direction: } T_{i} \cos \theta_{i}=T_{i-1} \cos \theta_{i-1}
\end{array}\right.
$$

\subsection{Analysis of the relevance of each component}

The total length of the projection of each member in the vertical direction should equal the depth of the seabed when the system is stable, which is ${ }^{h+\sum_{i=1}^{N} l_{i} \cos \theta_{i}=H}$. The radius of the floating circle of the buoy is $r=\sum_{i=1}^{N} l_{i} \sin \theta_{i}, \theta$ can be derived from the stress analysis in the previous text. 


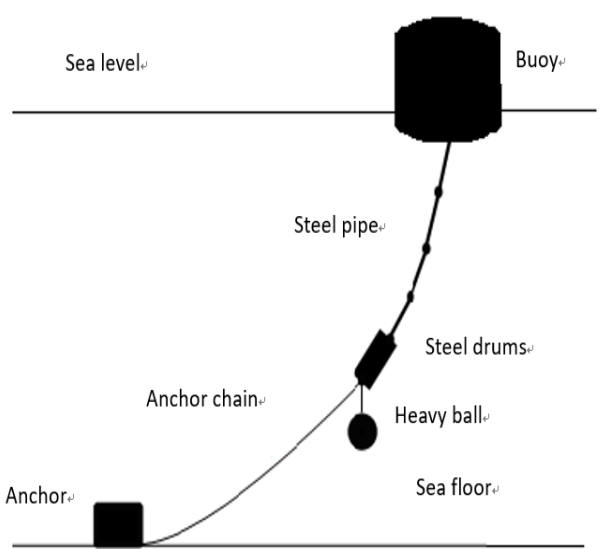

Figure 2. Schematic diagram of mooring system.

\section{4-Establishment of multi-objective model}

Taking the complex external conditions consideration, mooring system can still work properly, so properly designed mooring system parameters is indispensable, including the type of anchor $k$ and the heavy ball quality $m$. Therefore, a multi-objective optimization model is established. Optimization Goal 1: The depth $d$ of the buoy is affected only by the type $k$ of the chain, the length $s$ and the mass $m$ of the heavy ball, so it can be expressed as: $\min d(m, k, s)$. Optimization Goal 2: The area $r$ of the buoy is affected only by the type $k$ of the chain, the length $s$ and the mass $m$ of the heavy ball, so it can be expressed as: $\min r(m, k, s)$.

Create the following two constraints:

(1) the quality of heavy ball is indefinite and variable in a range, there are:

$$
M_{\text {min }}<m<M_{\text {max }}
$$

(2) The steel drum tilt angle can not exceed a certain degree, and the smaller the better, there are:

$$
0<\theta<\theta_{\max }
$$

\section{5-Mooring system optimization case analysis}

parameter

Select a type of mooring system [6] as an example of multi-objective optimization calculation. The basic parameters of the mooring system are as follows: Buoy system can be simplified as a cylinder, the bottom diameter and high of which both are $2 \mathrm{~m}$. The quality of the buoy is $1000 \mathrm{~kg}$. The quality of the anchor is $600 \mathrm{~kg}$. Common model and its parameters of near shallow sea observation are listed in table 1. There are four sections of steel pipe, one meter of each length, and the diameter is $50 \mathrm{~mm}$, and the quality of each steel tube is $10 \mathrm{~kg}$. The weight of the heavy ball is $1000 \mathrm{~kg}$ to $5000 \mathrm{~kg}$. The environmental parameters of the mooring system are as follows: the measured water depth of the sea area is $20 \mathrm{~m}$, the wind speed of the laying point is $36 \mathrm{~m} / \mathrm{s}$ and the sea water velocity is $1.5 \mathrm{~m} / \mathrm{s}$.
Table 1.Common anchor chain models and parameters

\begin{tabular}{ccc}
\hline Type & Length $(\mathbf{m m})$ & $\begin{array}{c}\text { Mass per unit length } \\
(\mathbf{k g} / \mathbf{m})\end{array}$ \\
\hline I & 78 & 3.2 \\
II & 105 & 7 \\
III & 120 & 12.5 \\
IV & 150 & 19.5 \\
V & 180 & 28.12 \\
\hline
\end{tabular}

The main control parameters of genetic algorithm are population number, times of iterations, hybrid rate, recombination rate and mutation rate. The final result and the efficiency of the genetic algorithm are affected by the population size. The small population will lead to the missed solution. The large population will increase the times of calculation of the adaptive function. The higher the crossover rate and the recombination rate are, the faster the individual updates in the population, but if too high, it will lead to the destruction of the outstanding individual. If too low, the quality of the population can not be further improved. The mutation rate cannot be too high, and if too high, the algorithm can continuously search the solution space and influence its convergence. When optimizing the design of mooring system parameters, the values of appropriate adjustment parameters are calculated through multiple trial calculations, and the relevant control parameters of genetic algorithm are shown in table2:

Table 2. genetic algorithm related parameters

\begin{tabular}{cc}
\hline Parameter & The numerical \\
\hline Population quantity & 200 \\
Number of iterations & 100 \\
Crossover rate & 0.9 \\
Number of objective functions & 2 \\
The mutation rate & 0.1 \\
Recombination rate & 0.7 \\
\hline
\end{tabular}

When using genetic algorithm to optimize the parameters of mooring system, a feasible solution can be obtained. The results of the improved crossover operator genetic algorithm and the results of the original genetic algorithm are shown in table 3 .

Table 3. Results comparison

\begin{tabular}{c|cc}
\hline \multicolumn{1}{|c}{$\begin{array}{c}\text { Improved } \\
\text { genetic } \\
\text { algorithm }\end{array}$} & $\begin{array}{c}\text { Primitive } \\
\text { genetic } \\
\text { algorithm }\end{array}$ \\
\hline Number of links & 161 & 230 \\
type & IV & II \\
Ball weight & 4601 & 4792 \\
Steel drum Angle & 4 & 3.4 \\
Winding radius & 19.71 & 22.43 \\
depth of immersion & 0.3 & 0.4 \\
\hline
\end{tabular}

Through the comparison of the above results, choose the type IV anchor chain. When the mass of the weight of the ball is $4601 \mathrm{~kg}$, the length of the chain buoy of the draft depth and the swimming area and the weight of the heavy ball can reach a lower value. The smaller the buoyancy depth is, the more obvious the buoy is, the smaller the swimming area is, the higher the stability of the whole system is. In this paper, the optimal design value of mooring system parameters obtained by 
improved crossover operator genetic algorithm has better effect on improving the stability of mooring system.

\section{6-Mooring system optimization case analysis}

parameter

The parameter optimization design of the mooring system is a multi - objective optimization problem with constraints. Using the genetic algorithm of improved crossover operator, the multi - objective optimization design of each parameter is solved. The result of the example shows that the optimized parameters obtained by this method can meet the constraints and improve the stability of mooring system. Therefore, this method can be carried out in multi-parameter optimization design effectively and provides a approach for other types of mooring system parameter optimization design.
1. Wang Mingwu. The static analysis and attitude calculation of the ocean submersible system [J]. Marine technology, 2001, (04) : 41-47.

2. Berteaux, H.O. Buoy Engineering. John Wiley \& Sons, New York, 1976

3. Li Zhihai, Xu Xingping, Wang Huili. Development of mooring system of offshore platform [J]. Oil field machinery, 2010, (05) : 75-78.

4. Xie Yanli, Xu Qinglin, Liang Wenchao. A genetic algorithm based on crossover and mutation operators [J]. Computer technology and development, 2014, (04) : 80-83.

5. Lu houqing, Chen liang, Song Yisheng, Wu Zhimin, Zou Yunbo. An improved algorithm for the crossover operator of genetic algorithm $[\mathrm{J}]$. Journal of PLA university of science and technology (natural science edition), 2007, (03) : 250-253.

6. national mathematical modeling contest for college students in 2016, question A, www.mcm.edu.cn/

\section{References}

